\title{
Type Synthesis of Parallel 2R1T Remote Center of Motion Mechanisms Based on Screw Theory
}

\author{
Long Huang, Chenhan Guang, Yang Yang and Peng Su \\ School of Mechanical Engineering and Automation, Beihang University, 100191 Beijing, China
}

\begin{abstract}
The remote center of motion (RCM) mechanism is an important part of a minimally invasive surgery (MIS) robot. As a practical type of RCM mechanisms, 2R1T RCM mechanisms are synthesized in this paper using a modified screw theory method. Differing from the conventional screw theory method, the modified method brings forward the assessment of different wrench systems, and proposes several general subchains to satisfy each geometrical condition of the wrench system, which can ensure the motion continuity to a great degree. First, the motion pattern and the twist system of 2R1T RCM mechanisms are presented. According to the reciprocal rules of screw theory, three corresponding wrench systems, along with the geometrical conditions for them, are enumerated. After assessing these wrench systems by the comparison of their geometrical conditions, the optimum wrench system is selected for synthesis, and then it is decomposed into sub-wrench-systems. To satisfy each condition of the subwrench-systems, several general subchains are proposed, and consequently some preferred general chains are constructed by the serial assembly of the corresponding subchains. These general chains are applied to the leg synthesis. Finally, practical examples of 2R1T RCM mechanisms are constructed by assembling the legs.
\end{abstract}

\section{Introduction}

Minimally invasive surgery (MIS) robots have attracted considerable attention in recent years [1-2]. In a MIS robot, the surgical tool should be capable of 3-DOF rotation around the incision and 1-DOF translation along the axis of the instrument. The remote center of motion (RCM) mechanism is particularly well suited for this. RCM mechanisms can be divided into six categories by DOF: 1-DOF rotation (1R), 2-DOF rotation (2R), 3-DOF rotation (3R), 1-DOF rotation and 1-DOF translation (1R1T), 2-DOF rotation and 1-DOF translation (2R1T), 3-DOF rotation and 1-DOF translation (3R1T). The multi-DOF RCM mechanism can be constructed by the serial assembly of RCM mechanisms with fewer DOFs. Taylor's group introduced the RCM mechanism to a laparoscopic robot [3-4]. Afterwards various kinds of RCM mechanisms have been reported, such as spherical mechanisms [5], arc-prismatic-joint RCM mechanisms [6], and parallelogram-based RCM mechanisms [7]. Also, several methods, such as the virtual chain method and the module combination method, are proposed for the type synthesis of some specific kinds of RCM mechanisms [810]. However, for multi-DOF mechanisms, a widely used type synthesis method is the screw theory method. The major disadvantage of this method is that it can only synthesize instantaneous motion mechanisms [11-14].

As a practical type of RCM mechanisms, 2R1T RCM mechanisms are synthesized in this paper using a modified screw theory method. First, the twist system of
2R1T RCM mechanisms are presented, and the corresponding wrench system with the simplest conditions are selected and decomposed into sub-wrenchsystems. Section 5 concentrates on the leg synthesis. In this section, the conditions for each wrench are analyzed in detail, and each condition can be satisfied by a serial of newly-constructed subchains; by assembling the corresponding subchains, some general chains which satisfy all the conditions are obtained. On this basis, legs with each sub-wrench-system are synthesized. Finally in Section 6, the examples of 2R1T RCM mechanisms are constructed by assembling the corresponding legs. Compared with the conventional screw theory based synthesis processes, this paper brings forward the assessment of different wrench systems, and proposes several general subchains for each geometrical condition, which can ensure the motion continuity of the mechanisms to a large degree.

\section{Type synthesis method based on screw theory}

Based on the line geometry, screw theory can be used to express the motion and constraint of a mechanism, and these two aspects of a mechanism are connected through the reciprocal rules. In screw theory, the motion of a mechanism is represented by a twist system which is composed of several twists, and the constraint for a mechanism is represented by a wrench system which is 
composed of several wrenches. The details about screw theory can be seen in [11-13].

Assuming that a twist system (or wrench system) contains $n$ independent non-redundant twists (or wrenches), then the corresponding wrench system (or twist system) contains (6- $n$ ) independent non-redundant wrenches (or twists). Furthermore, the general reciprocal rules and type synthesis processes in screw theory can be found in [11-12]. However, owing to the particularity of the RCM motion pattern, the processes should be adapted for the specific synthesis problems.

In this paper, twists and wrenches of pitch $h$ are represented by $\$^{\text {h }}$ and $\hat{\$}^{\text {h }}$ respectively. Twist systems of order $\mathrm{n}$ formed by zero pitch twists (or infinite pitch twists) are denoted by $\mathrm{n}-\$^{0}$ (or $\mathrm{n}-\$^{\infty}$ ), and wrench systems of order $\mathrm{n}$ formed by zero pitch wrenches (or infinite pitch wrenches) are denoted by $\mathrm{n}-\hat{\$}^{0}$ (or $\left.\mathrm{n}-\hat{\$}^{\infty}\right)$. Twist systems and wrench systems can be illustrated in graph [12]: zero pitch twists and wrenches are represented by lines, while infinite pitch twists and wrenches are represented by arrows.

\section{Preparation for type synthesis}

The output link of the 2R1T RCM mechanism can tilt and roll around the incision point, and translate along the axis of the instrument. Assuming the instrument is mounted on the moving platform of the mechanism with the axis perpendicular to the plane of the moving platform. The base frame $\left\{\mathrm{F}_{\mathrm{b}}\right\}$ and the moving platform frame $\left\{\mathrm{F}_{\mathrm{m}}\right\}$ are shown in Fig. 1. Then the moving platform of the $2 \mathrm{R} 1 \mathrm{~T}$ RCM mechanism can rotate about the origin of $\left\{F_{b}\right\}$ and translate along the $\mathrm{z}_{\mathrm{m}}$-axis in $\left\{\mathrm{F}_{\mathrm{m}}\right\}$.

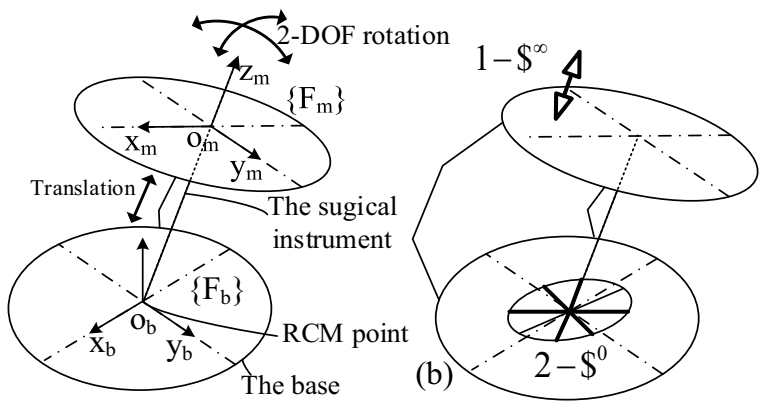

Figure 1. (a) Coordinate frame definition of the motion pattern; (b) the line graph representation of twist system.

According to the motion pattern of the mechanism, the required twist system for it is derived to be $2-\$^{0}-1-\$^{\infty}$. To realize the required motion pattern, $2-\$^{0}-1-\$^{\infty}$ system should satisfy the following conditions at each configuration of the given workspace: (1) The axis of $\$^{\infty}$ should be parallel to the $z_{m}$-axis; (2) The center of $2-\$^{0}$ should be the origin of $\left\{\mathrm{F}_{\mathrm{b}}\right\}$, and the plane of $2-\$^{0}$ should not be coplanar with the $\mathrm{z}_{\mathrm{m}}$-axis. The line graph representation of the twist system is shown in Fig. 1(b).

\section{The wrench systems of the 2R1T RCM mechanism}

According to the reciprocal rules, the wrench system of the mechanism is a 3 -system, which is generated by three independent wrenches. Three wrench systems corresponding to the twist system are shown in Fig. 2.

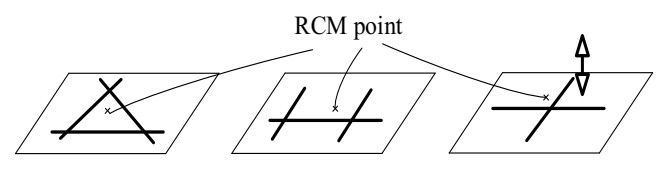

(a)

(b)

(c)

Figure 2. The line graph representation of three wrench systems Wrench systems in Fig. 2a and Fig. 2b are both $3-\hat{\$}^{0}$ systems. According to the geometrical conditions for the twist system and the reciprocal rules, the wrenches in these systems should fulfill the following conditions.

Condition (a1): three zero pitch wrenches should be coplanar; they either form a triangle on a plane, or two of them are parallel and intersect the rest wrench.

Condition (a2): the plane generated by the wrenches should be parallel to the plane of the moving platform. Condition (a3): The RCM point should lie on the plane generated by the wrenches.

Wrench system in Fig. $2 \mathrm{c}$ is a $2-\hat{\$}^{0}-1-\hat{\$}^{\infty}$ system. The wrenches in this system should fulfill the following conditions.

Condition (c1): two zero pitch wrenches should intersect. The direction of the infinite pitch wrench $\hat{\$}^{\infty}$ should not be parallel to the plane generated by $2-\hat{\$}^{0}$.

Condition (c2): the plane generated by $2-\hat{\$}^{0}$ should be parallel to $x_{m} y_{m}$-plane.

Condition (c3): The RCM point should lie on the plane generated by $2-\hat{\$}^{0}$.

For each one of these wrench systems, if all the listed conditions are satisfied at any configuration in a certain area, then the corresponding mechanisms can maintain the motion pattern in this area. However, not every condition can be satisfied easily. Through the comparison of the conditions for these wrench systems, we can find the optimum wrench system for synthesis. First, each one of these three wrench systems contains two zero pitch wrenches, and the conditions for them are basically the same. And evidently coplanarity (Condition (a3)) is a much stronger condition than non-parallelism (Condition (c2)). If we choose wrench systems $3-\hat{\$}^{0}$, it is likely that we will obtain 2R1T RCM mechanisms with bifurcated motion or parasitic motion [9]. Hence, we choose wrench system $2-\hat{\$}^{0}-1-\hat{\$}^{\infty}$ to synthesize 2R1T RCM mechanisms. This wrench system can be decomposed in many ways, and each part of it corresponds to a single leg. All possible parts of the wrench system can be listed as follows: $2-\hat{\$}^{0}-1-\hat{\$}^{\infty}$, $1-\hat{\$}^{0}-1-\hat{\$}^{\infty}, 2-\hat{\$}^{0}, 1-\hat{\$}^{0}$ and $1-\hat{\$}^{\infty}$. The following 
paragraphs deal with the synthesis of legs corresponding to each of these sub-wrench-systems.

\section{Synthesis of legs}

Unlike the conventional screw theory method, the modified method regards the processes of leg synthesis as the key to the guarantee of motion continuity. Based on the condition analysis of each wrench, we can translate each condition into a class of subchains, and furthermore assemble the subchains into general chains. For clarity of explanation, we adopt the visual line graph to represent the wrench system, instead of mathematical equations.

\subsection{Condition analysis}

Condition (c1) claims that the two zero pitch wrenches should intersect. For convenience of leg synthesis, the intersecting point is designated as the RCM point. In order to ensure the zero pitch wrenches of a leg passing through a fixed point at every configuration, an effective way is to install a subchain consisting of two or more revolute joints intersecting at a fixed point in the leg (the subchain is denoted by $(\mathrm{R} . . . \mathrm{R})_{\mathrm{S}}$ or $\underline{\mathrm{R}}$... $\left.\underline{\mathrm{R}}\right)$. Since there are only three independent twists (or wrenches) among all of the concurrent twists (or wrenches) in space [11], the required non-redundant subchains can be of two types: (1) $(\mathrm{RR})_{\mathrm{S}}$, and (2) (R...R $)_{\mathrm{S}}$, as Fig. 3a and 3b shows; furthermore, to keep the intersecting point fixed while the configuration changes, $(\mathrm{RR})_{\mathrm{S}}$-subchain or $(\mathrm{R} \ldots \mathrm{R})_{\mathrm{S}}$ subchain in the leg should be mounted as close to the base as possible. In addition, there are a class of chains containing two intersecting revolute joints, yet these joints are separated by other joints, for instance, the RRRR -subchain shown in Fig. 3c. In order to keep the axes of the first joint and the last joint intersecting at a fixed point, there should be some additional constraint generated by other legs, which will be discussed later.
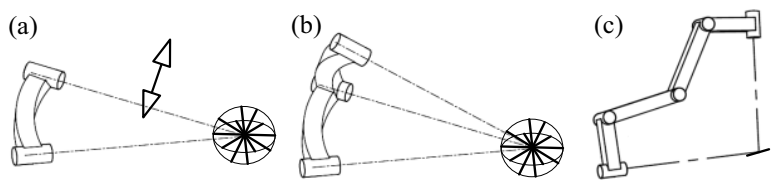

Figure 3. Subchains with intersecting axes.

Condition (c2) says each zero pitch wrench should be parallel to the $x_{m} y_{m}$-plane. There are two methods for this condition according to the reciprocal rules: (1) the leg includes a prismatic joint with its axis perpendicular to the $\mathrm{x}_{\mathrm{m}} \mathrm{y}_{\mathrm{m}}$-plane; (2) the leg includes a subchain consisting of two or more parallel revolute joints whose axes are parallel to the $\mathrm{x}_{\mathrm{m}} \mathrm{y}_{\mathrm{m}}$-plane in the leg (this subchain is denoted by $(\mathrm{R} . . . \mathrm{R})_{\mathrm{E}}$ or $\left.\overline{\mathrm{R}} . . . \overline{\mathrm{R}}\right)$. Since there are only three independent parallel twists (or wrenches) among all of the spatial parallel twists (or wrenches), the required non-redundant parallel subchains can be of two types: (1) $(R R)_{E}$, and (2) $(R R R)_{E}$. One thing to note is that each one of these two types has a class of variants. Revolute joints in $(\mathrm{R} . . . \mathrm{R})_{\mathrm{E}}$-subchain can be replaced by prismatic joints partially without influencing the wrench system, as long as the subchain is still a planar mechanism. Fig. 4 shows some examples of $(R R)_{E}$-type and $(R R R)_{E}$-type. Furthermore, in order to guarantee the parallel relationship while the configuration changes, the prismatic joint or $(\mathrm{R} . . . \mathrm{R})_{\mathrm{E}}$-subchain in the leg should be mounted as close to the moving platform as possible.
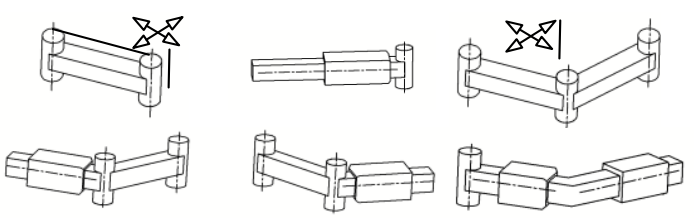

Figure 4. Subchains of $(R R)_{E}$-type and $(R R R)_{E}$-type

Condition (c3) says that the direction of the infinite pitch wrench $\hat{\$}^{\infty}$ is not parallel to the plane generated by $2-\hat{\$}^{0}$, which is automatically satisfied on almost every occasion. Therefore condition (c3) can be ignored during the leg synthesis.

In conclusion, during the leg synthesis process, we give preference to the following general serial chains: $(\mathrm{R} \ldots \mathrm{R})_{\mathrm{S}} \ldots(\mathrm{R} \ldots \mathrm{R})_{\mathrm{E}}, \quad(\mathrm{R} \ldots \mathrm{R})_{\mathrm{S}} \ldots \mathrm{P}$ and $\underline{\mathrm{R}} \ldots(\mathrm{R} \ldots \mathrm{R})_{\mathrm{E}} \mathrm{R}$. First, we apply the preferred chains to the specific situation so that we can obtain chains corresponding to the required wrench system without any geometric conditions. Then, in terms of all geometric conditions for the wrench system, the qualified chains are selected and the assembly conditions for them are obtained.

\subsection{Synthesis of legs corresponding to sub- wrench-system $2-\hat{\$}^{0}-1-\hat{\$}^{\infty}$}

For a non-redundant serial kinematic chain corresponding to sub-wrench-system $2-\hat{\$}^{0}-1-\hat{\$}^{\infty}$, the order of it is three. Therefore, the serial chain has three joints according to the reciprocal rules. In fact, the corresponding chain is actually a serial $2 \mathrm{R} 1 \mathrm{~T}$ RCM mechanism.

Applying the preferred chains to the situation, we only obtain the $(\mathrm{RR})_{\mathrm{S}} \mathrm{P}$-chain (Fig. 5). The serial chain shown in Fig. 5a is the simplest chain with the required sub-wrench-system. The kinematic chain shown in Fig. $5 \mathrm{~b}$ is constructed through serial connection of a revolute joint, an arc prismatic joint and a prismatic joint. It can also be considered as a $(\mathrm{RR})_{\mathrm{S}} \mathrm{P}$-chain. According to the reciprocal rules, the rest of the assembly conditions for the joints are: (1) the axes of the first two joints should intersect at the appointed RCM point; (2) the axis of the prismatic joint should be parallel to the $\mathrm{z}_{\mathrm{m}}$-axis.

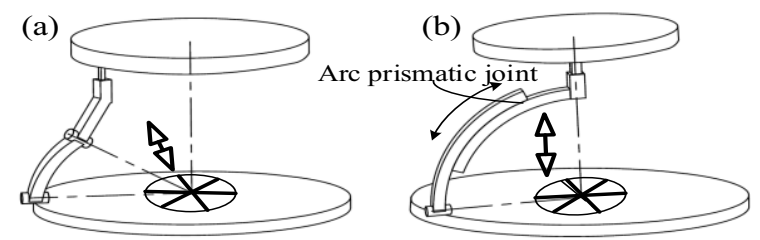

Figure 5. Serial kinematic chains with $2-\hat{\$}^{0}-1-\hat{\$}^{\infty}$ 


\subsection{Synthesis of legs corresponding to sub- wrench-system $1-\hat{\$}^{0}-1-\hat{\$}^{\infty}$}

A non-redundant serial chain with sub-wrench-system $1-\hat{\$}^{0}-1-\hat{\$}^{\infty}$ has four joints. Applying the preferred chains to the situation, we obtain two possible types: $(R R)_{S}(R R)_{E}$-type and $R(R R R)_{E}$-type.

For $(R R)_{S}(R R)_{E}$-type, the sub-wrench-system requires that the axes of revolute joints in $(R R)_{E}$ subchain be parallel to the plane generated by the axes of $(R R)_{S}$. Therefore, the possible serial chains include: $(R \bar{R})_{S} \bar{R} \bar{R},(R \bar{R})_{S} \bar{P} \bar{R},(R \bar{R})_{S} \bar{R} \bar{P}$ and $(R \bar{R})_{S} \bar{P} \bar{P}$. Other than the internal geometrical relationships, these chains should also satisfy the following conditions: (1) the intersecting point of $(R R)_{S}$-subchain should be the RCM point, and (2) the axis of $\bar{R} \bar{R} \bar{R}$-subchain should be parallel to the $\mathrm{x}_{\mathrm{m}} \mathrm{y}_{\mathrm{m}}$-plane.

For $R(R R R)_{E}$-type, there are following possible chains: $R(R R R)_{E}, R(R P R)_{E}, R(P R R)_{E}, R(R R P)_{E}$, $\mathrm{R}(\mathrm{RPP})_{\mathrm{E}}, \mathrm{R}(\mathrm{PRP})_{\mathrm{E}}, \mathrm{R}(\mathrm{PPR})_{\mathrm{E}}$. The additional assembly conditions for them are: (1) the axis of the first revolute joint should pass the RCM point; (2) the axis of $(\mathrm{RRR})_{\mathrm{E}}$-subchain should be parallel to the $\mathrm{x}_{\mathrm{m}} \mathrm{y}_{\mathrm{m}}$-plane; (3) the adjacent axes of the two subchains should be set skewed. Fig. 6 shows two examples of $(R R)_{S}(R R)_{E}-$ type and $R(R R R)_{E}$-type.
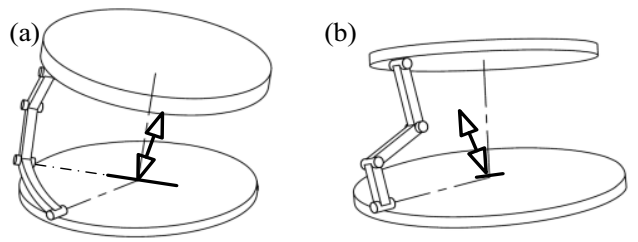

Figure 6. Two legs corresponding to $1-\hat{\$}^{0}-1-\hat{\$}^{\infty}$

\subsection{Synthesis of legs corresponding to sub- wrench-system $2-\hat{\$}^{0}$}

A non-redundant serial chain corresponding to subwrench-system $2-\widehat{\$}^{0}$ also contains four joints. Since the geometrical conditions focus on the two zero pitch wrenches, there are few qualified serial chains. After verifying each one of the preferred general chains, we only obtain the $(\mathrm{RRR})_{\mathrm{S}} \mathrm{P}$ chain. Two examples are shown in Fig. 7. Each leg consists of three revolute joints intersecting at the RCM point and a prismatic joint perpendicular to the platform.

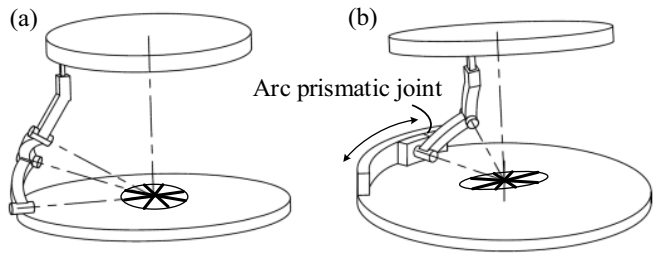

Figure 7. Two examples of $(\mathrm{RRR})_{\mathrm{S}} \mathrm{P}$-chain

\subsection{Synthesis of legs corresponding to sub- wrench-system $1-\hat{\$}^{0}$}

The conditions for sub-wrench-system $1-\hat{\$}^{0}$ are listed as follows: (1) the wrench should be parallel to the $\mathrm{x}_{\mathrm{m}} \mathrm{y}_{\mathrm{m}}{ }^{-}$ plane, and (2) it should pass the RCM point. A nonredundant serial chain corresponding to sub-wrenchsystem $1-\hat{\$}^{0}$ contains five joints. Applying the preferred serial chains to the situation, we can obtain three following types which satisfy the geometric conditions for the sub-wrench-system: $(R R R)_{S}(R R)_{E}$-type, $(R R)_{S}(R R R)_{E}$-type and $\underline{R}(R R R)_{E} \underline{R}$-type.

For $(R R R)_{S}(R R)_{E}$-type, there are following possible chains: $(\mathrm{RRR})_{\mathrm{S}}(\mathrm{RR})_{\mathrm{E}},(\mathrm{RRR})_{\mathrm{S}}(\mathrm{PR})_{\mathrm{E}},(\mathrm{RRR})_{\mathrm{S}}(\mathrm{RP})_{\mathrm{E}}$, and $(R R R)_{S}(P P)_{E}$. For $(R R)_{S}(R R R)_{E}$-type, there are $(R R)_{\mathrm{S}}(\mathrm{RRR})_{\mathrm{E}} \quad, \quad(\mathrm{RR})_{\mathrm{S}}(\mathrm{RRP})_{\mathrm{E}} \quad, \quad(\mathrm{RR})_{\mathrm{S}}(\mathrm{RPR})_{\mathrm{E}}$ ， $(\mathrm{RR})_{\mathrm{S}}(\mathrm{PRR})_{\mathrm{E}}, \quad(\mathrm{RR})_{\mathrm{S}}(\mathrm{RPP})_{\mathrm{E}}, \quad(\mathrm{RR})_{\mathrm{S}}(\mathrm{PRP})_{\mathrm{E}}$ and $(\mathrm{RR})_{\mathrm{S}}(\mathrm{PPR})_{\mathrm{E}}$. The additional conditions for them are: (1) the intersecting point of $(\mathrm{R} . . . \mathrm{R})_{\mathrm{S}}$-subchain should be the RCM point; (2) the axis of $(\mathrm{R} . . . R)_{\mathrm{E}}$-type subchain should be parallel to the $\mathrm{x}_{\mathrm{m}} \mathrm{y}_{\mathrm{m}}$-plane; (3) the adjacent axes of the two subchains should be set skewed.

For $\underline{R}(R R R)_{E} \underline{R}$-type, there are following possible chains: $\underline{R}(R R R)_{E} \underline{R} ， \quad \underline{R}(R P R)_{E} \underline{R} ， \underline{R}(R R P)_{E} \underline{R}$ ， $\underline{\mathrm{R}}(\mathrm{PRR})_{\mathrm{E}} \underline{\mathrm{R}}, \underline{\mathrm{R}}(\mathrm{PPR})_{\mathrm{E}} \underline{\mathrm{R}}, \underline{\mathrm{R}}(\mathrm{RPP})_{\mathrm{E}} \underline{\mathrm{R}}$ and $\underline{\mathrm{R}}(\mathrm{PRP})_{\mathrm{E}} \underline{\mathrm{R}}$.

Fig. 8 shows four examples of these three types.
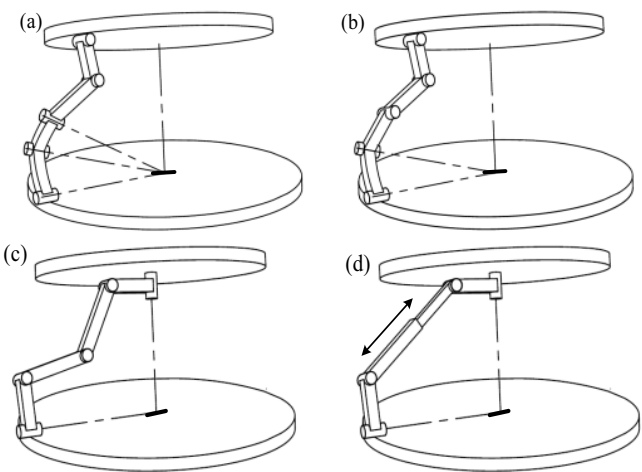

Figure 8. Four legs corresponding to sub-wrench-system $1-\hat{\$}^{0}$ : (a) $(\mathrm{RRR})_{\mathrm{S}}(\mathrm{RR})_{\mathrm{E}}$-type, (b) $(\mathrm{RR})_{\mathrm{S}}(\mathrm{RRR})_{\mathrm{E}}$-type, (c) $\underline{\mathrm{R}}(\mathrm{RRR})_{\mathrm{E}} \underline{\mathrm{R}}$ and (d) $\underline{\mathrm{R}}(\mathrm{RPR})_{\mathrm{E}} \underline{\mathrm{R}}$.

\subsection{Synthesis of legs corresponding to sub- wrench-system $1-\hat{\$}^{\infty}$}

Condition (c3) brings in little constraint on the wrenches. Hence, there is no need to apply the preferred serial chain in this section. Two typical types are $(R R R)_{E}(R R)_{E}-$ type and $(\mathrm{RR})_{\mathrm{E}}(\mathrm{RRR})_{\mathrm{E}}$-type [11]. For both types, the assembly conditions are listed as follows: (1) the adjacent axes of the two subchains should be set skewed, and (2) the axes of both subchains should not be perpendicular to the $\mathrm{x}_{\mathrm{m}} \mathrm{y}_{\mathrm{m}}$-plane. 


\section{Synthesis of 2R1T RCM mechanisms}

On the basis of leg synthesis, several practical mechanisms with required motion pattern can be obtained. As mentioned before, only 3-legged parallel mechanisms are considered. For convenience of expression, these three legs are denoted by leg I, leg II and leg III. Using combinatorial method, the possible types of nonredundant 2R1T RCM mechanisms are listed in Table 1.

Table 1. The combination of three legs corresponding to the given sub-wrench-systems.

\begin{tabular}{|c|c|c|c|}
\hline & Leg I & Leg II & Leg III \\
\hline Type (i) & $2-\hat{\$}^{0}-1-\hat{\$}^{\infty}$ & $\varnothing$ & $\varnothing$ \\
\hline Type (ii) & $1-\hat{\$}^{0}-1-\hat{\$}^{\infty}$ & $1-\hat{\$}^{0}$ & $\varnothing$ \\
\hline Type (iii) & $2-\hat{\$}^{0}$ & $1-\hat{\$}^{\infty}$ & $\varnothing$ \\
\hline Type (iv) & $1-\hat{\$}^{\infty}$ & $1-\hat{\$}^{\infty}$ & $1-\hat{\$}^{\infty}$ \\
\hline
\end{tabular}

The concrete mechanisms of these types can be constructed by simply assembling the corresponding legs, and some examples are shown in Table 2. It is easy to verify the motion continuity of these mechanisms.

Table 2. Examples of the four types and a practical prototype.

Type (ii)

\section{Conclusions}

As an important kind of RCM mechanisms, the 2R1T RCM mechanisms are synthesized using a modified screw theory method. By following standard procedures, the twist system and several wrench systems of $2 \mathrm{R} 1 \mathrm{~T}$ RCM mechanisms are obtained. Then, this paper brings forward discussion of the synthesis difficulties, which contributes to the selection of the optimum wrench system. Afterwards, the wrench system is decomposed and synthesized respectively. During the processes, some preferred serial chains are proposed to meet the geometrical conditions, which can ensure the motion continuity of mechanisms to a great extent. Finally, several practical examples of $2 \mathrm{R} 1 \mathrm{~T}$ RCM mechanisms are constructed by assembling the corresponding legs. Some of them can be translated into practical prototypes.

\section{References}

1. A. Gijbels, E.B. Vander Poorten, P. Stalmans, H. Van Brussel, Design of a teleoperated robotic system for retinal surgery, IEEE ICRA, 2357-2363 (2014).

2. G.S. Guthart, J.K. Salisbury, The Intuitive telesurgery system: overview and application, IEEE ICRA, 618 - 621 (2000).

3. R.H. Taylor, J. Funda, B. Eldridge, S. Gomory, A telerobotic assistant for laparoscopic surgery. IEEE Eng. Med. Biol. Mag. 14, 279-288 (1995).

4. R.H. Taylor, D. Stoianovici, Medical robotics in computer-integrated surgery. IEEE Trans. on Rob. Autom 19, 765-781(2003).

5. L. Huang, Y. Yang, P Su, J Xiao, Type Synthesis of 1R1T Remote Center of Motion Mechanisms. $J$. Mech. Eng 13, 131-136 (2015). (in Chinese)

6. T. Ueta, Y. Yamaguchi, Y. Shirakawa, T. Nakano, R. Ideta, Y. Noda, Robot-assisted vitreoretinal surgery: development of a prototype and feasibility studies in an animal model. Ophthalmology 116, 1-2 (2009).

7. M.J.H. Lum, D.C.W. Friedman, H. King, The RAVEN: Design and Validation of a Telesurgery System. Int. J. Robot. Res. 28 (2009) 1183-1197.

8. G. Zong, X. Pei, J. Yu, S. Bi, Classification and type synthesis of 1-DOF remote center of motion mechanisms. Mech. Mach. Theory. 43, 1585-1595 (2008).

9. J. Li, G. Zhang, A. Müller, S. Wang, A Family of Remote Center of Motion Mechanisms Based on Intersecting Motion Planes. J. Mech. Design 135 , 091009-1-10 (2013).

10. X. Kong, C.M. Gosselin, Type synthesis of 4-DOF SP-equivalent parallel manipulators: A virtual chain approach. Mech. Mach. Theory 41 1306-1319 (2006).

11. Z. Huang, Q. Li, H. Ding, Theory of Parallel Mechanisms. Mech. Mach. Science. 6, 653-4 (2013).

12. F. Xie, X.J. Liu, T. Li, Type Synthesis and Typical Application of 1T2R-Type Parallel Robotic Mechanisms. Math. Probl. Eng. 6, 497-504 (2013).

13. N. Kumar, O. Piccin, B. Bayle, A task-based type synthesis of novel 2T2R parallel mechanisms. Mech. Mach. Theory. 77, 59-72 (2014).

14 P. S. Rao, N. M. Rao Position Analysis of Spatial 3RPS Parallel Manipulator. Int. J. Mech. Eng. \& Rob. Res. 2, 80-90 (2013). 